Case Report

\title{
Effective Administration of Rituximab in Anti-MDA5 Antibody-Positive Dermatomyositis with Rapidly Progressive Interstitial Lung Disease and Refractory Cutaneous Involvement: A Case Report and Literature Review
}

\author{
Yuka Ogawa, ${ }^{1}$ Dai Kishida, ${ }^{1}$ Yasuhiro Shimojima, ${ }^{1}$ Koichi Hayashi, ${ }^{2}$ and Yoshiki Sekijima $^{1}$ \\ ${ }^{1}$ Department of Medicine (Neurology and Rheumatology), Shinshu University School of Medicine, 3-1-1 Asahi, Matsumoto \\ 390-8621, Japan \\ ${ }^{2}$ Department of Dermatology, Shinshu University School of Medicine, 3-1-1 Asahi, Matsumoto 390-8621, Japan
}

Correspondence should be addressed to Yasuhiro Shimojima; yshimoji@shinshu-u.ac.jp

Received 31 July 2017; Accepted 24 September 2017; Published 31 October 2017

Academic Editor: Gregory J. Tsay

Copyright (C) 2017 Yuka Ogawa et al. This is an open access article distributed under the Creative Commons Attribution License, which permits unrestricted use, distribution, and reproduction in any medium, provided the original work is properly cited.

\begin{abstract}
We describe the case of a 48-year-old man with dermatomyositis (DM) who demonstrated rapidly progressive interstitial lung disease (RP-ILD) and refractory cutaneous involvement together with high levels of anti-melanoma differentiation-associated gene 5 antibody (anti-MDA5-Ab). Even after combination immunosuppressive therapy including a corticosteroid, cyclosporine $\mathrm{A}$, and intravenous cyclophosphamide, his respiratory insufficiency and cutaneous involvement progressively worsened. However, the administration of rituximab (RTX) resulted in clinical remission as well as a visible reduction in anti-MDA5-Ab levels, suggesting that RTX could be a useful remedy in cases refractory to conventional immunosuppressive agents, especially those of RP-ILD related to anti-MDA5-Ab-positive DM.
\end{abstract}

\section{Introduction}

Dermatomyositis (DM) is an autoimmune inflammatory myopathy with characteristic cutaneous involvement such as Gottron's papules, a heliotrope rash, and/or an erythematous eruption around the neck and shoulders [1]. As a subtype of DM, clinically amyopathic DM (CADM), which is characterized by a typical skin lesion of DM with no or subclinical muscular manifestations, is substantially differentiated from classic DM [2]. In both CADM and classic DM, interstitial lung disease (ILD) has been recognized as the complication impacting on the prognosis; moreover, numerous studies have recently described that the development of rapidly progressive ILD (RP-ILD) is implicated in the positivity of anti-melanoma differentiation-associated gene 5 antibody (anti-MDA5-Ab), which is more frequently detected in patients with CADM than in those with classic DM [3-6].

Although combination immunosuppressive therapy consisting of a corticosteroid, calcineurin inhibitor, and intravenous cyclophosphamide (IVCY) is sometimes selected to prevent patients with DM-related RP-ILD from developing fatal disease, such an intensive therapeutic strategy is not entirely sufficient to ensure a favorable prognosis [7-9]. Rituximab (RTX), a chimeric monoclonal antibody for depleting B cells showing CD20 protein, was recently demonstrated to be effective for intractable muscular and/or cutaneous involvement in polymyositis or DM $[10,11]$. It was also suggested that RTX could be useful for severe ILD in antisynthetase syndrome $[12,13]$; meanwhile, there are only a few case reports in which RTX was used in anti-MDA5-Abpositive DM with ILD [14-18].

Here we describe a case in which RTX ameliorated RP-ILD as well as refractory cutaneous involvement in a patient with anti-MDA5-Ab-positive DM despite the resistance to the conventional immunosuppressive therapy. We also review the literature for studies of RTX in anti-MDA5$\mathrm{Ab}$-positive DM with ILD. 


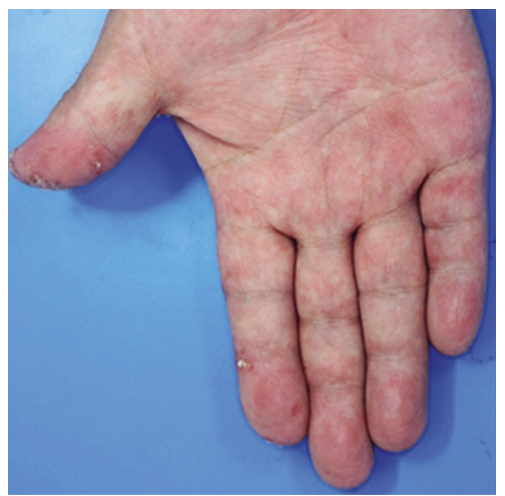

(a)

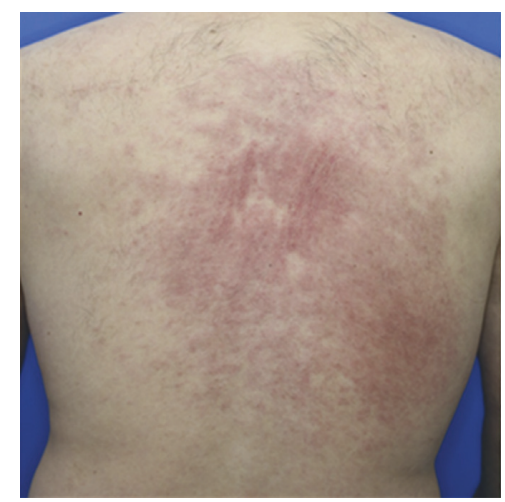

(b)

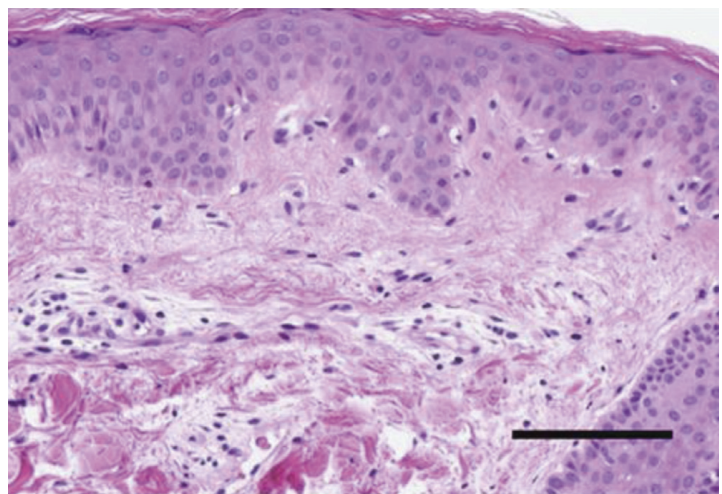

(c)

FIGURE 1: Skin lesions including palmar papules (a) and erythema on the back (b) before the initiation of treatment. A skin biopsy from the back indicates liquefactive degeneration with perivascular inflammation between the epidermis and dermis (c) (hematoxylin and eosin staining; scale bar $=100 \mu \mathrm{m}$ ).

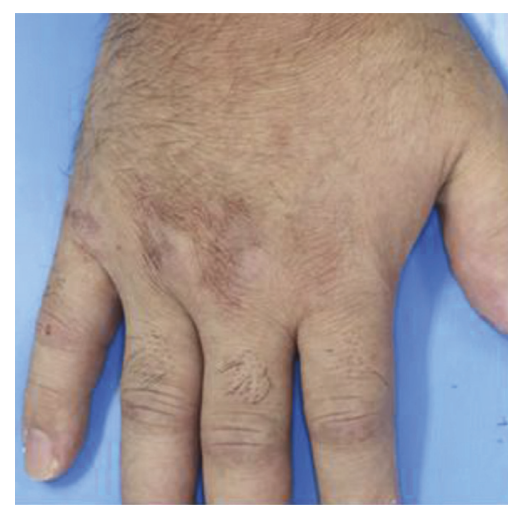

(a)

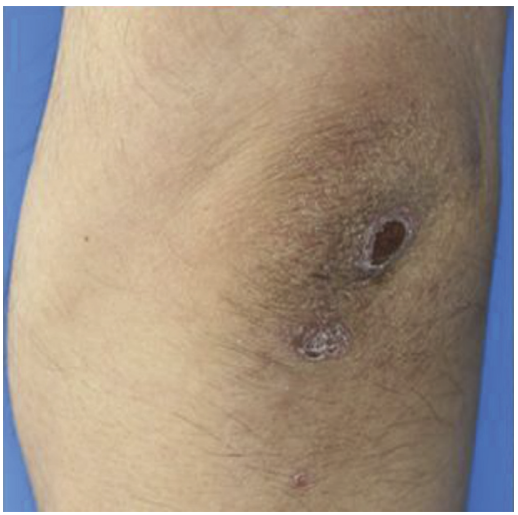

(d)

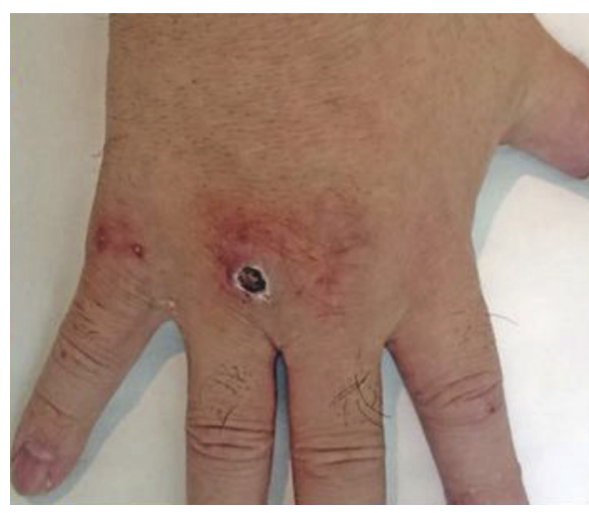

(b)

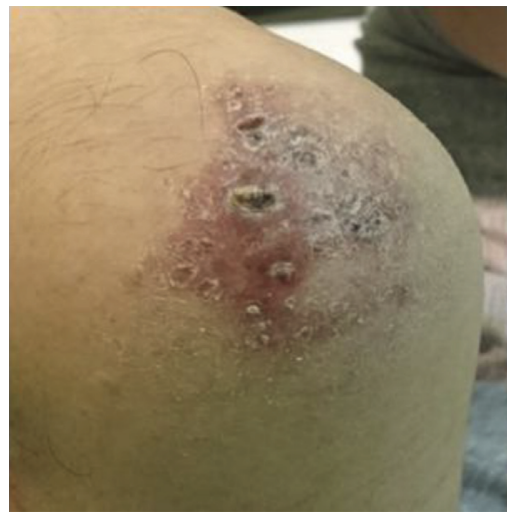

(e)

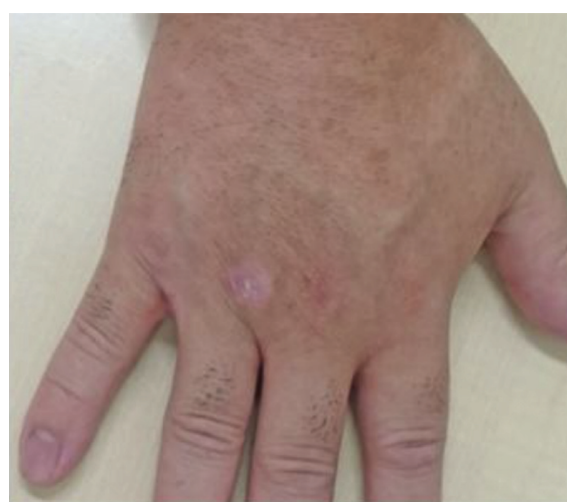

(c)

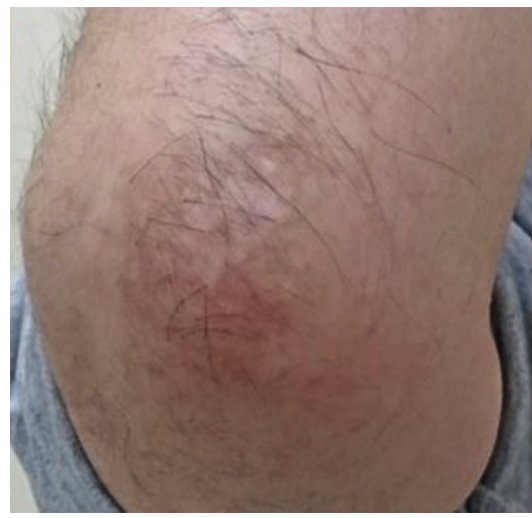

(f)

Figure 2: Sequential findings of cutaneous lesions on the dorsum of the hand and elbow before the initiation of treatment (a, d), before the addition of rituximab (RTX) (b, e), and after RTX administration (c, f).

\section{Case Presentation}

A 48-year-old man with a 1-month history of fatigue, appetite loss, and fever was admitted to our hospital. He reported experiencing arthralgia and a dry cough as well as mild exertional dyspnea prior to admission. A physical examination demonstrated a body temperature of $37.7^{\circ} \mathrm{C}$, mild muscular weakness of the proximal lower limbs, edematous hands, and cutaneous manifestations including a heliotrope rash, Gottron's papules, mechanic's hands, 


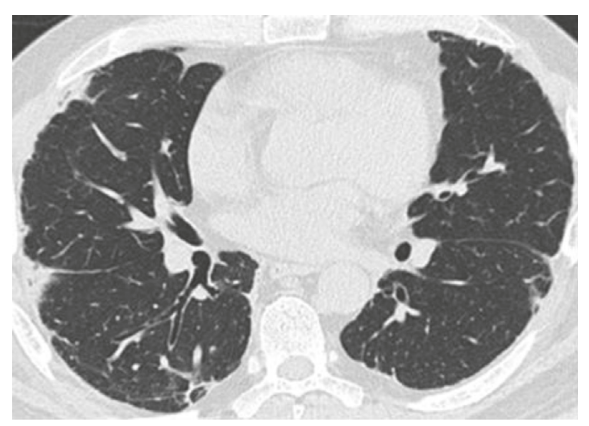

(a)

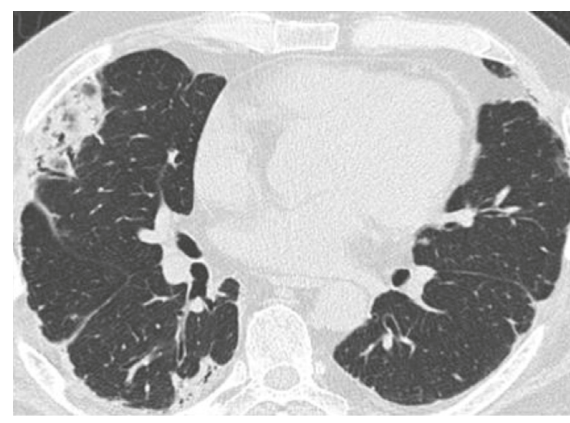

(b)

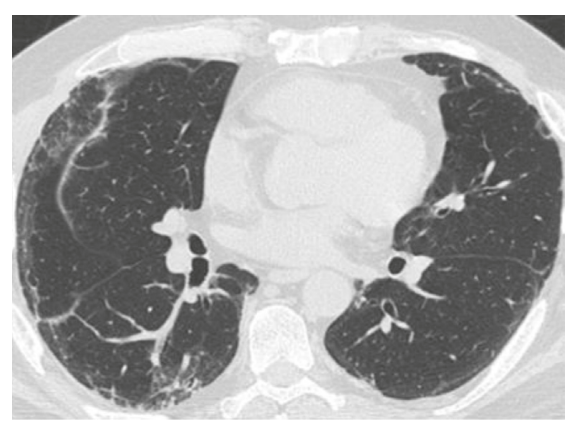

(c)

Figure 3: Chest computed tomography findings at admission (a), before the addition of rituximab (RTX) (b), and after RTX administration (c).
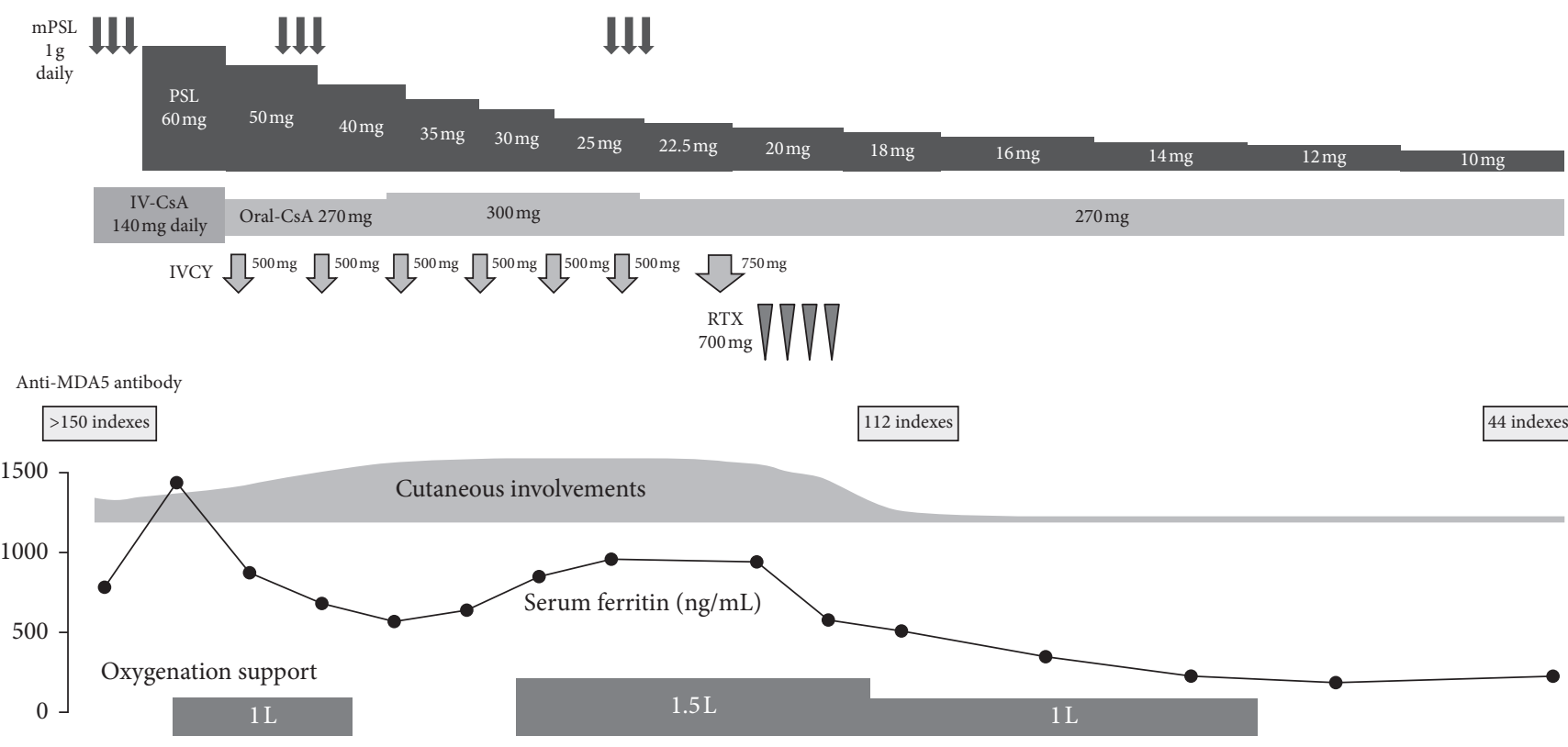

FIgURE 4: Clinical course of this patient. mPSL, methylprednisolone; PSL, prednisolone; IV-CsA, continuous intravenous infusion of cyclosporine A; oral-CsA, oral administration of cyclosporine A; IVCY, intravenous infusion of cyclophosphamide; RTX, rituximab.

palmar papules, and an erythematous rash on his face and back; in particular, ulcerative and erosive erythema was visible on his elbows (Figures 1 and 2). The pathological finding of the biopsied skin from his back demonstrated perivascular infiltration of inflammatory cells with liquefactive degeneration through the epidermis to the dermis (Figure 1(c)). Laboratory examinations revealed elevated serum levels of creatine kinase $(\mathrm{CK})(278 \mathrm{U} / \mathrm{L}$; normal, $43-230)$, C-reactive protein $(0.59 \mathrm{mg} / \mathrm{dL}$; normal, $<0.10)$, ferritin $(781 \mathrm{ng} / \mathrm{mL}$; normal, 25-280), Krebs von den Lungen-6 (602 U/mL; normal, 105-435), and lactate dehydrogenase (453 U/L; normal, 120-230). Anti-aminoacyltRNA synthetase (anti-ARS) antibodies were not detected; meanwhile, a high titer of anti-MDA5-Ab was seen $(>150$ indexes; normal, <32). The detection of anti-MDA5-Ab was performed by enzyme-linked immunosorbent assay. Tests for anti-nuclear antibody, rheumatoid factor, anti-citrullinated protein antibody, and anti-neutrophil cytoplasmic antibodies specific for either myeloperoxidase or proteinase- 3 were negative. Arterial blood gas analysis revealed a $\mathrm{PaO}_{2}$ of $66.4 \mathrm{mmHg}$ and $\mathrm{PaCO}_{2}$ of $32.9 \mathrm{mmHg}$ on room air. Chest computed tomography (CT) revealed reticular shadows and ground-glass opacity on the middle to inferior fields of the bilateral lung (Figure 3(a)).

Since the patient was diagnosed with DM-related ILD and a low $\mathrm{PaO}_{2}$, methylprednisolone (mPSL) (1 g daily for 3 consecutive days) was immediately administered together with a continuous intravenous infusion of cyclosporine A (IV-CsA) according to the previously described therapeutic protocol [9] (Figure 4). Subsequently, prednisolone (PSL) at the dose of $60 \mathrm{mg}(1 \mathrm{mg} / \mathrm{kg})$ daily and cyclosporine $\mathrm{A}$, the blood trough concentration of which was adjusted to $150-200 \mathrm{ng} / \mathrm{mL}$, were orally administered after mPSL and IV-CsA therapy, resulting in improved fatigue, appetite loss, fever, edema of the hands, eruptions on the face and back, and arthralgia. Muscle weakness also recovered, and serum $\mathrm{CK}$ level normalized. Meanwhile, respiratory insufficiency persisted, and oxygenation support was ultimately required. 


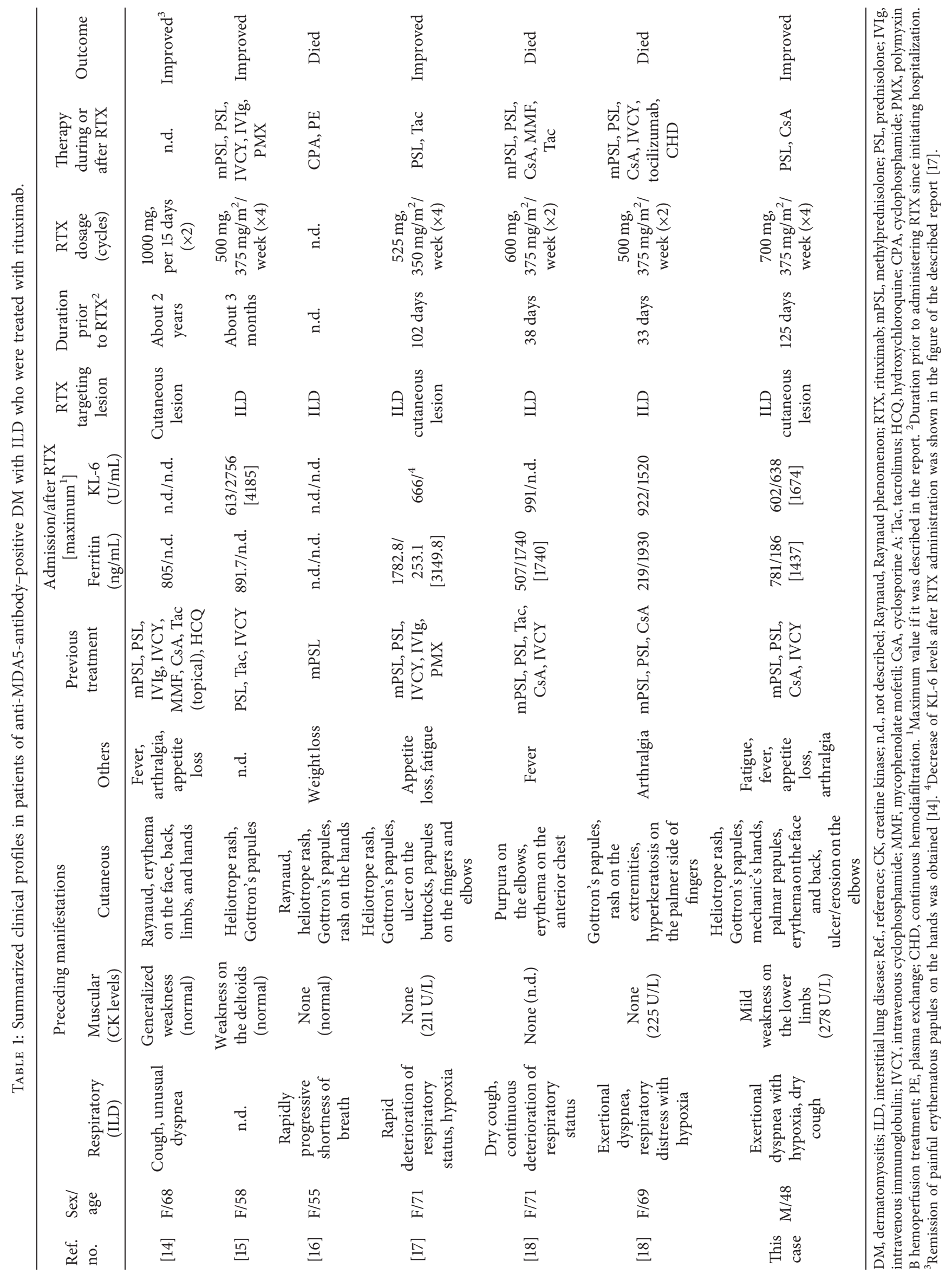


Therefore, IVCY was additionally administered at the dosage of $500 \mathrm{mg}$ every 2 weeks for 6 cycles. However, his respiratory status was gradually worsening even after additional administration of $750 \mathrm{mg}$ of IVCY. Moreover, further exacerbation of the chest CT finding was obviously demonstrated (Figure 3(b)). In addition, ulcerative and erosive eruptions on his hands and elbows also deteriorated (Figures 2(b) and $2(\mathrm{e}))$. On the 125 th day since the initiation of the therapy, RTX was administered at the dosage of $700 \mathrm{mg}\left(375 \mathrm{mg} / \mathrm{m}^{2}\right)$ weekly for total 4 weeks. Consequently, cutaneous involvements on his hands and elbows were remarkably improved (Figures 2(c) and 2(f)), and recovery from respiratory insufficiency and amelioration of the radiographic finding were also achieved (Figure 3(c)). Furthermore, serum levels of ferritin and anti-MDA5-Ab decreased to $186 \mathrm{ng} / \mathrm{mL}$ and 44 indexes, respectively (Figure 4).

\section{Discussion}

This patient was found to be in the severe stage of ILD associated with DM because of having some prognostic factors including not only hypoxemia with anti-MDA5-Ab positivity but also refractory ulcerative eruptions as well as palmer papules. In fact, it has been previously described that existing cutaneous ulceration and/or palmer papules are associated with acute progression of ILD in DM [6, 7]; furthermore, the positive correlation between severity of ulcerative cutaneous involvement and anti-MDA5-Ab has been demonstrated $[6,19,20]$. In addition, anti-MDA5-Ab positivity is associated with fever and arthritis $[19,20]$, which were also revealed prior to initiating treatment in the present case. Therefore, immediate and intensive immunosuppressive therapy was required in this patient in order to avoid a fatal situation. It has been recognized that the concomitant use of CsA or tacrolimus with PSL is indispensable to prevent the progression of ILD in DM [21-23]; furthermore, additional administration of IVCY should be required in the acute exacerbation of the disease [7, 9, 23]. However, initial combination therapy, including PSL, CsA, and IVCY, was eventually insufficient for healing both cutaneous involvement and ILD. On the other hand, RTX therapy obviously contributed to achieving a favorable outcome.

MDA5 plays a crucial role in inducing an innate immune response against viral infection and is also recognized as the specific autoantigen in DM, suggesting that upregulation of MDA5 in the innate immune system subsequently promotes anti-MDA5-Ab production [24]. The production of specific autoantibodies in autoimmune diseases is attributed to autoreactive B cells; furthermore, RTX is found to succeed in depleting the pathogenic autoreactive B cells which secrete a specific autoantibody in several autoimmune diseases [25]. Even though autoreactive $\mathrm{B}$ cells also play the roles in activating effector $\mathrm{T}$ cells or producing proinflammatory cytokines, RTX may be indirectly implicated in preventing these immunological factors from attacking the target organ [25-27]. Interestingly, the recent overviewed study demonstrated that favorable therapeutic efficacy of RTX could be obtained in majority of patients with inflammatory myositis who had disease-specific autoantibodies [28]. It was described that high titer of anti-MDA5-Ab is associated with the severity of disease $[29,30]$. On the other hand, subsequent reduction of anti-MDA5-Ab after starting the treatment means obviously predicting a successful outcome [20]. Although this patient initially had a level of anti-MDA5-Ab over the upper measurable limit, RTX administration reduced the levels of anti-MDA5-Ab and serum ferritin, which is also known as the prognostic factor in DM-related RP-ILD [31] (Figure 4), demonstrating that RTX therapy could correspondingly contribute to the recovery from severe clinical situations and suppress the production of anti-MDA5-Ab. Given the relationship between the therapeutic mechanism of RTX and autoantibodies including anti-MDA5-Ab, RTX therapy may be a reasonable option for achieving a favorable outcome, especially in RP-ILD related to anti-MDA5-Ab-positive DM.

To our knowledge, six cases treated with RTX in antiMDA5-Ab-positive DM with ILD have been reported in the English literature to date [14-18] (Table 1). Their clinical characteristics were CADM or minimal muscular manifestations, the latter of which is consistent with the present case. One patient was also given RTX to treat the refractory cutaneous involvement even after ILD remission induced by the prior immunosuppressive therapy, whereas others required RTX because the preceding use of other immunosuppressive agents was ineffective in suppressing the exacerbation of ILD with or without cutaneous involvement. However, only half of them recovered from the RP-ILD, suggesting that it may be necessary to initiate effective therapy within the reversible state of disease even if RTX is a potential remedy in cases that are resistant to the prevalent immunosuppressive therapies.

Anti-MDA5-Ab-positive DM usually emerges with refractory cutaneous involvement and/or RP-ILD as a lifethreatening complication. The present case demonstrated that RTX could be a useful therapy for achieving a favorable outcome. On the other hand, further clinical experiences must be accumulated to establish the therapeutic strategy using RTX for this disease.

\section{Conflicts of Interest}

The authors declare no financial or personal conflicts of interest.

\section{References}

[1] M. C. Dalakas and R. Hohlfeld, "Polymyositis and dermatomyositis," Lancet, vol. 362, no. 9388, pp. 971-982, 2003.

[2] P. Gerami, J. M. Schope, L. McDonald, H. W. Walling, and R. D. Sontheimer, "A systematic review of adult-onset clinically amyopathic dermatomyositis (dermatomyositis sine myositis): a missing link within the spectrum of the idiopathic inflammatory myopathies," Journal of the American Academy of Dermatology, vol. 54, no. 4, pp. 597-613, 2006.

[3] S. Sato, M. Hirakata, M. Kuwana et al., "Autoantibodies to a 140-kd polypeptide, CADM-140, in Japanese patients with clinically amyopathic dermatomyositis," Arthritis and Rheumatism, vol. 52, no. 5, pp. 1571-1576, 2005.

[4] T. Gono, Y. Kawaguchi, T. Satoh et al., "Clinical manifestation and prognostic factor in anti-melanoma differentiation-associated 
gene 5 antibody-associated interstitial lung disease as a complication of dermatomyositis," Rheumatology, vol. 49, no. 9, pp. 1713-1719, 2010.

[5] Y. Muro, K. Sugiura, K. Hoshino, and M. Akiyama, "Disappearance of anti-MDA-5 autoantibodies in clinically amyopathic DM/interstitial lung disease during disease remission," Rheumatology, vol. 51, no. 5, pp. 800-804, 2012.

[6] H. Cao, M. Pan, Y. Kang et al., "Clinical manifestations of dermatomyositis and clinically amyopathic dermatomyositis patients with positive expression of anti-melanoma differentiationassociated gene 5 antibody," Arthritis Care \& Research, vol. 64, no. 10, pp. 1602-1610, 2012.

[7] H. Kameda, H. Nagasawa, H. Ogawa et al., "Combination therapy with corticosteroids, cyclosporin $\mathrm{A}$, and intravenous pulse cyclophosphamide for acute/subacute interstitial pneumonia in patients with dermatomyositis," Journal of Rheumatology, vol. 32, no. 9, pp. 1719-1726, 2005.

[8] Y. Matsuki, H. Yamashita, Y. Takahashi et al., "Diffuse alveolar damage in patients with dermatomyositis: a six-case series," Modern Rheumatology, vol. 22, no. 2, pp. 243-248, 2012.

[9] Y. Shimojima, W. Ishii, M. Matsuda, D. Kishida, and S. I. Ikeda, "Effective use of calcineurin inhibitor in combination therapy for interstitial lung disease in patients with dermatomyositis and polymyositis," Journal of Clinical Rheumatology, vol. 23, no. 2, pp. 87-93, 2017.

[10] C. V. Oddis, A. M. Reed, R. Aggarwal et al., "Rituximab in the treatment of refractory adult and juvenile dermatomyositis and adult polymyositis: a randomized, placebo-phase trial," Arthritis and Rheumatism, vol. 65, no. 2, pp. 314-324, 2013.

[11] R. Aggarwal, P. Loganathan, D. Koontz, Z. Qi, A. M. Reed, and C. V. Oddis, "Cutaneous improvement in refractory adult and juvenile dermatomyositis after treatment with rituximab," Rheumatology, vol. 56, no. 2, pp. 247-254, 2017.

[12] H. Andersson, M. Sem, M. B. Lund et al., "Long-term experience with rituximab in anti-synthetase syndrome-related interstitial lung disease," Rheumatology, vol. 54, no. 8, pp. 1420-1428, 2015.

[13] I. Marie, S. Dominique, A. Janvresse, H. Levesque, and J. F. Menard, "Rituximab therapy for refractory interstitial lung disease related to antisynthetase syndrome," Respiratory Medicine, vol. 106, no. 4, pp. 581-587, 2012.

[14] A. Clottu, E. Laffitte, C. Prins, and C. Chizzolini, "Response of mucocutaneous lesions to rituximab in a case of melanoma differentiation antigen 5-related dermatomyositis," Dermatology, vol. 225, no. 4, pp. 376-380, 2012.

[15] R. Watanabe, T. Ishii, K. Araki et al., "Successful multi-target therapy using corticosteroid, tacrolimus, cyclophosphamide, and rituximab for rapidly progressive interstitial lung disease in a patient with clinically amyopathic dermatomyositis," Modern Rheumatology, vol. 26, no. 3, pp. 465-466, 2016.

[16] B. Gil, L. Merav, L. Pnina, and G. Chagai, "Diagnosis and treatment of clinically amyopathic dermatomyositis (CADM): a case series and literature review," Clinical Rheumatology, vol. 35, no. 8, pp. 2125-2130, 2016.

[17] Y. Koichi, Y. Aya, U. Megumi et al., "A case of anti-MDA5positive rapidly progressive interstitial lung disease in a patient with clinically amyopathic dermatomyositis ameliorated by rituximab, in addition to standard immunosuppressive treatment," Modern Rheumatology, vol. 27, no. 3, pp. 536-540, 2017.

[18] K. Tokunaga and N. Hagino, "Dermatomyositis with rapidly progressive interstitial lung disease treated with rituximab: a report of 3 cases in Japan," Internal Medicine, vol. 56, no. 11, pp. 1399-1403, 2017.
[19] D. Fiorentino, L. Chung, J. Zwerner, A. Rosen, and L. CasciolaRosen, "The mucocutaneous and systemic phenotype of dermatomyositis patients with antibodies to MDA5 (CADM-140): a retrospective study," Journal of the American Academy of Dermatology, vol. 65, no. 1, pp. 25-34, 2011.

[20] S. Sato, A. Murakami, A. Kuwajima et al., "Clinical utility of an enzyme-linked immunosorbent assay for detecting antimelanoma differentiation-associated gene 5 autoantibodies," PLoS One, vol. 11, no. 4, p. e0154285, 2016.

[21] T. Kotani, S. Makino, T. Takeuchi et al., "Early intervention with corticosteroids and cyclosporin A and 2-hour postdose blood concentration monitoring improves the prognosis of acute/subacute interstitial pneumonia in dermatomyositis," Journal of Rheumatology, vol. 35, no. 2, pp. 254-259, 2008.

[22] T. Kurita, S. Yasuda, K. Oba et al., "The efficacy of tacrolimus in patients with interstitial lung diseases complicated with polymyositis or dermatomyositis," Rheumatology, vol. 54, no. 1, pp. 39-44, 2015.

[23] T. Kurita, S. Yasuda, O. Amengual, and T. Atsumi, "The efficacy of calcineurin inhibitors for the treatment of interstitial lung disease associated with polymyositis/dermatomyositis," Lupus, vol. 24, no. 1, pp. 3-9, 2015.

[24] S. Sato, K. Hoshino, T. Satoh et al., "RNA helicase encoded by melanoma differentiation-associated gene 5 is a major autoantigen in patients with clinically amyopathic dermatomyositis: association with rapidly progressive interstitial lung disease," Arthritis and Rheumatism, vol. 60, no. 7, pp. 21932200, 2009.

[25] P. Engel, J. A. Gomez-Puerta, M. Ramos-Casals, F. Lozano, and X. Bosch, "Therapeutic targeting of B cells for rheumatic autoimmune diseases," Pharmacological Reviews, vol. 63, no. 1, pp. 127-156, 2011.

[26] R. Stasi, G. Del Poeta, E. Stipa et al., "Response to B-cell depleting therapy with rituximab reverts the abnormalities of T-cell subsets in patients with idiopathic thrombocytopenic purpura," Blood, vol. 110, no. 8, pp. 2924-2930, 2007.

[27] N. Jacob and W. Stohl, "Autoantibody-dependent and autoantibody-independent roles for B cells in systemic lupus erythematosus: past, present, and future," Autoimmunity, vol. 43, no. 1, pp. 84-97, 2010.

[28] S. Fasano, P. Gordon, R. Hajji, E. Loyo, and D. A. Isenberg, "Rituximab in the treatment of inflammatory myopathies: a review," Rheumatology, vol. 56, no. 1, pp. 26-36, 2017.

[29] T. Takada, A. Aoki, K. Asakawa et al., "Serum cytokine profiles of patients with interstitial lung disease associated with antiCADM-140/MDA5 antibody positive amyopathic dermatomyositis," Respiratory Medicine, vol. 109, no. 9, pp. 1174-1180, 2015.

[30] S. Sato, M. Kuwana, T. Fujita, and Y. Suzuki, "Anti-CADM140/MDA5 autoantibody titer correlates with disease activity and predicts disease outcome in patients with dermatomyositis and rapidly progressive interstitial lung disease," Modern Rheumatology, vol. 23, no. 3, pp. 496-502, 2013.

[31] T. Gono, Y. Kawaguchi, M. Hara et al., "Increased ferritin predicts development and severity of acute interstitial lung disease as a complication of dermatomyositis," Rheumatology, vol. 49, no. 7, pp. 1354-1360, 2010. 


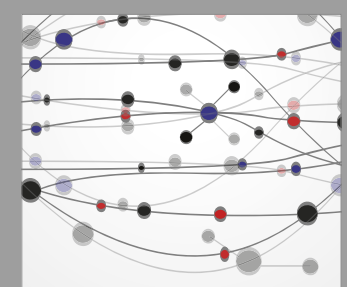

The Scientific World Journal
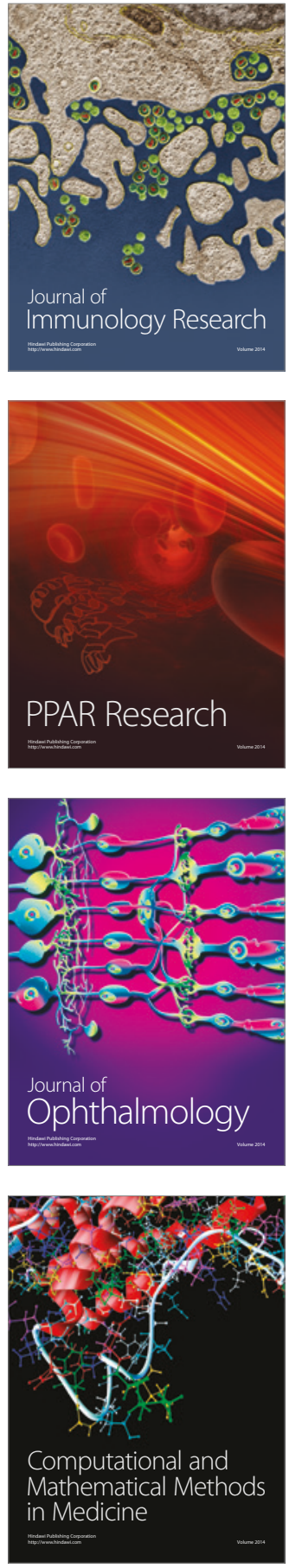

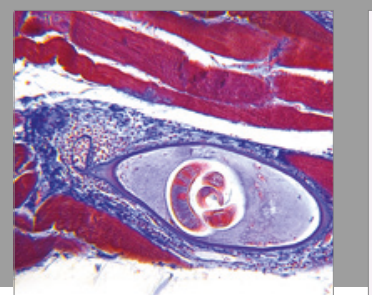

Gastroenterology Research and Practice
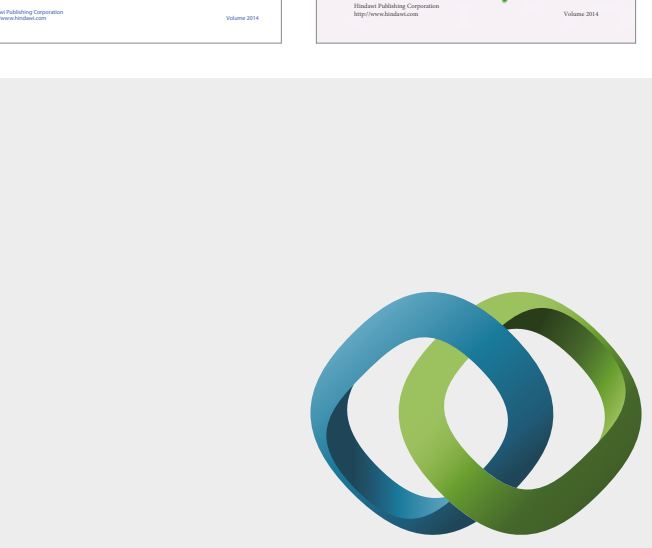

\section{Hindawi}

Submit your manuscripts at

https://www.hindawi.com
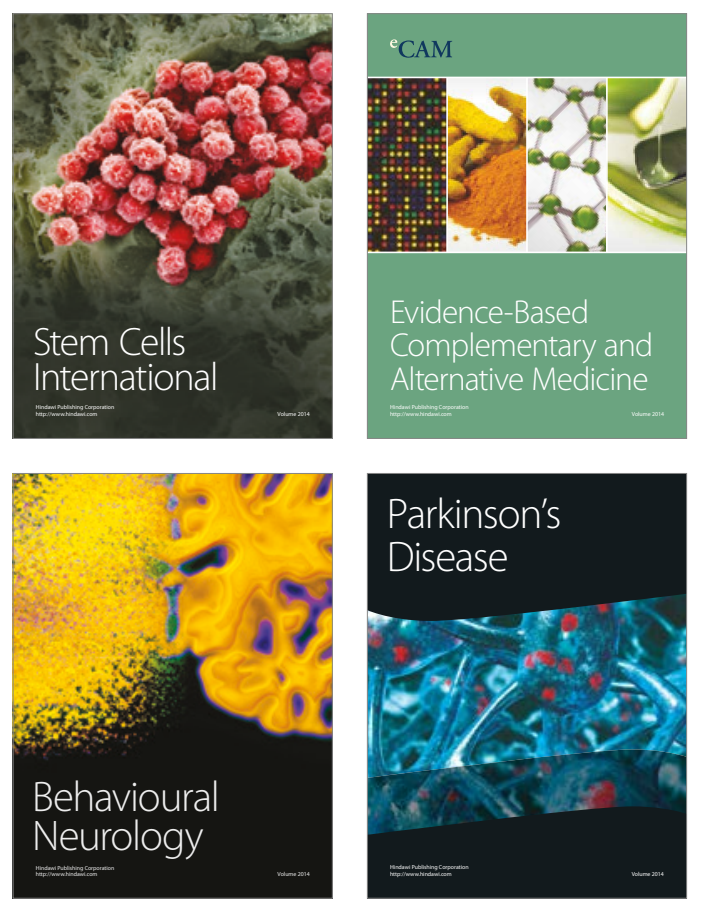
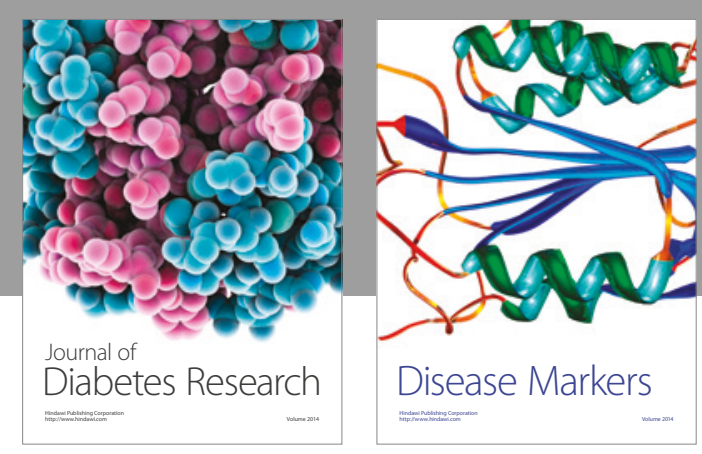

Disease Markers
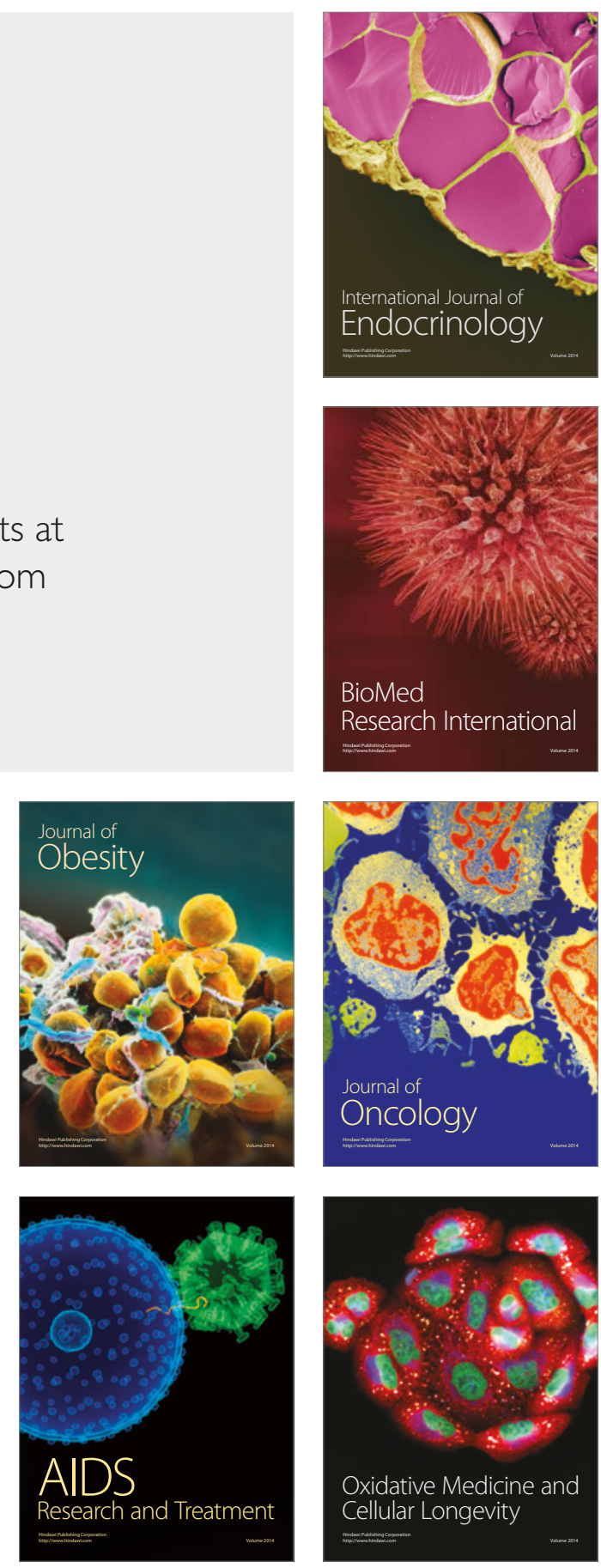